\title{
ON A THEOREM CONCERNING EXISTENCE OF INTERPOLATING FUNCTIONS $\left({ }^{1}\right)$
}

\author{
BY \\ RICHARD F. DEMAR
}

In a recent paper [4], the author proved a theorem concerning the existence of a function in a class of entire functions of exponential type which interpolates a given sequence of complex numbers relative to a given sequence of linear functionals. We show here that the hypothesis that a certain set is convex may be weakened. Cases in which the new form of the theorem is needed are then shown.

1. Modification of the theorem. Let $K$ be the set of all entire functions of exponential type and let $T=\left\{\mathscr{L}_{n}\right\}$ be a sequence of linear functionals defined on $K$ by

$$
\mathscr{L}_{n}(f)=\frac{1}{2 \pi i} \int_{\Gamma}[W(\zeta)]^{n} F(\zeta) d \zeta
$$

for some function $W$ regular at the origin with $W(0)=0$ and $W^{\prime}(0) \neq 0$ where $F$ is the Borel (Laplace) transform of $f$ and $\Gamma$ is a simple contour enclosing all singularities of $F$. If $G$ is an open connected subset of the plane, we use $K[G]$ to denote the set of all $f$ in $K$ such that all singularities of $F$ are contained in $G$. We say that a sequence $\left\{b_{n}\right\}$ of complex numbers is admissible for $T$ and $K[G]$ if and only if there exists a function $f \in K[G]$ such that $\mathscr{L}_{n}(f)=b_{n}(n=0,1,2, \cdots)$. If the only function in $K[G]$ such that $\mathscr{L}_{n}(f)=0(n=0,1,2, \ldots)$ is $f=0$, then $K[G]$ is called a uniqueness class for $T$. Buck [2] proved that if $W$ is regular and univalent on an open connected set $\Omega_{\zeta}$ containing the origin whose image $\Omega_{w}$ under $w=W(\zeta)$ is star shaped with respect to the origin, then $K\left[\Omega_{\zeta}\right]$ is a uniqueness class for $T$.

For $\Omega_{\zeta}$ satisfying the above conditions, the author [4] proved sufficiency in the following admissibility theorem.

Theorem 1. Let $T=\left\{\mathscr{L}_{n}\right\}$ have a representation (1.1). Let $C_{\zeta}$ be an open convex subset of $\Omega_{\zeta}$ containing the origin. Then a necessary and sufficient condition that a sequence $\left\{b_{n}\right\}$ of complex numbers be admissible for the transformation $T$ and the class $K\left[C_{5}\right]$ is that $b(z)=\sum_{n=0}^{\infty} b_{n} z^{n}$ be regular at the origin and have a continuation analytic on $1 / C_{w}^{\prime}$, the complement of the image of $C_{\zeta}$ under the map $t=1 / W(\zeta)$.

Received by the editors July 1, 1963.

$\left({ }^{1}\right)$ A part of this work was done during the author's tenure of a National Academy of SciencesNational Research Council Post-doctoral Resident Research Associateship at the National Bureau of Standards while on leave from Miami University. 
Buck [3] had proved necessity without the hypothesis that $C_{5}$ be convex. We now show that this hypothesis can be weakened to simple connectedness. To prove sufficiency, given a sequence $\left\{b_{n}\right\}$ of complex numbers such that $b(z)$ (defined by $\sum_{n=0}^{\infty} b_{n} z^{n}$ and its analytic continuation) is regular on $1 / C_{w}^{\prime}$, a function $f$ was defined by

$$
f(z)=\frac{1}{2 \pi i} \int_{E} \frac{b(t)}{t} e^{z W^{-1}(1 / t)} d t
$$

where $E$ is a simple contour enclosing $1 / C_{w}^{\prime}$ and is contained in a simply connected region of regularity of $b$ and $W^{-1}$ is the inverse of $W$ which maps $\Omega_{w}$ onto $\Omega_{5}$. It was then shown that $\mathscr{L}_{n}(f)=b_{n}(n=0,1,2, \ldots)$. The convexity of $C_{5}$ was used only in showing that the set of singularities of $F$ was contained in $C_{\zeta}$, i.e., that $f \in K\left[C_{\zeta}\right]$. We now show that $f \in K\left[C_{\zeta}\right]$ without assuming that $C_{\zeta}$ is convex, but only that it is simply connected.

The Borel transform $F$ of $f$ is given by

$$
F(\zeta)=\int_{0}^{\infty} e^{-\zeta s} f(s) d s
$$

in a half plane $\mathscr{R}(\zeta)>k$ for some $k$. For such $\zeta$, we have

$$
\begin{aligned}
F(\zeta) & =\int_{0}^{\infty} e^{-\zeta s}\left[\frac{1}{2 \pi i} \int_{E} \frac{b(t)}{t} e^{s W^{-1}(1 / t)} d t\right] d s \\
& =\frac{1}{2 \pi i} \int_{E} \frac{b(t)}{t}\left[\int_{0}^{\infty} e^{-\zeta s} e^{s W^{-1}(1 / t)} d s\right] d t
\end{aligned}
$$

where the interchange of the order of integration is justified by the existence of the integral

$$
\int_{E} \frac{|b(t)|}{|t|} \int_{0}^{\infty}\left|e^{-\zeta s} e^{s W-1(1 / t)}\right| d s d t
$$

But wherever the integral $\int_{0}^{\infty} e^{-\zeta s} e^{s W^{-1}(1 / t)} d s$ exists it is the Laplace transform of $e^{s W^{-1}(1 / t)}$ which is $1 /\left(\zeta-W^{-1}(1 / t)\right)$, so substituting this function in (1.2) gives the desired analytic continuation of $F$; i.e.

$$
F(\zeta)=\frac{1}{2 \pi i} \int_{E} \frac{b(t)}{t\left(\zeta-W^{-1}(1 / t)\right)} d t
$$

for all $\zeta$ for which this integral exists. Thus $F$ is analytic at least for all $\zeta$ for which for all $t$ on $E, \zeta \neq W^{-1}(1 / t)$. But $E$ is contained in $1 / C_{w}$, the image of $C_{\zeta}$ under $t=1 / W(\zeta)$. Therefore, if $\zeta \in C_{\zeta}^{\prime}$, the complement of $C_{\zeta}$, then for all $t$ on $E, \zeta \neq W^{-1}(1 / t)$. Therefore $F$ is regular on $C_{\zeta}^{\prime}$ or all of the singularities of $F$ are in $C_{\zeta}$; i.e., $f \in K\left[C_{\zeta}\right]$. Thus the hypothesis that $C_{\zeta}$ is convex is unnecessary. 
2. Difference functionals with complex $\beta$. In the special cases of transformations $T$ having a representation (1.1) which have been most commonly studied (e.g., Newton, Stirling, and Abel functionals), the function $W$ is an entire function. To find as large a uniqueness class $K\left[\Omega_{\zeta}\right]$ as possible, one determines a domain $\Omega_{\zeta}$ containing the origin on which $W$ is univalent whose image $\Omega_{\zeta}$ under the map $W$ is star shaped with respect to the origin and such that $W$ is not univalent on any open set $G$ which properly contains $\Omega_{5}$. Then $K[G]$ is not a uniqueness class for $T$ since $W\left(a_{1}\right)=W\left(a_{2}\right)$ implies $\mathscr{L}_{n}\left(e^{a_{1} z}\right)=$ $\mathscr{L}_{n}\left(e^{a_{2} z}\right)(n=0,1,2, \ldots)[5]$. In applying Theorem 1 , one of the most interesting choices of the set $C_{\zeta}$ is the whole set $\Omega_{\zeta}$. In most special cases studied, a set $\Omega_{\zeta}$ determined in the manner described above is convex, so that the admissibility theorem as previously proved could be applied to obtain an admissibility condition for $T$ and $K\left[\Omega_{\zeta}\right]$. In fact, the author knows of no published results in which this is not the case. We give now a class of transformations $T$ for which sets $\Omega_{\zeta}$ are not convex.

Let $\left\{\mathscr{L}_{n}\right\}$ be the sequence of functionals given by

$$
\mathscr{L}_{n}(f)=\Delta^{n} f(\beta n)=\sum_{k=0}^{n}(-1)^{n+k}\left(\begin{array}{l}
n \\
k
\end{array}\right) f(\beta n+k)
$$

where $\beta$ is a parameter. These have a representation (1.1) with $W(\zeta)=$ $e^{\beta \zeta}\left(e^{\zeta}-1\right)$. Uniqueness classes have been given for $\beta \geqq 0, \beta \leqq-1$, and $\beta=$ $-1 / 2\left[1\right.$, p. 34]. In all of these cases $\Omega_{\zeta}$ is convex. We study certain cases of complex $\beta$ with nonzero imaginary part and obtain nonconvex $\Omega_{5}$.

If $\beta$ is real, then $\beta n+k$ is real for all integers $n$ and $k$, so that the differences $\Delta^{n} f(\beta n)$ are determined entirely by the behavior of $f$ on the real axis. If $\beta$ is nonreal, then $\beta n+k(n=0,1,2, \cdots ; k=0,1, \cdots, n)$ lie in the closed sector bounded by $\theta=\arg \beta$ and $\theta=\arg (\beta+1)$. Thus the values of $\Delta^{n} f(\beta n)$ are influenced by the values of $f$ at points distributed over this sector. For example, if $\beta=i, \beta n+k(n=0,1,2, \cdots ; k=0,1, \cdots, n)$ are the lattice points in the closed sector with boundary $\theta=\pi / 4$ and $\theta=\pi / 2$. From this one might expect larger uniqueness classes for nonreal $\beta$ than for real $\beta$, but this is not the case. For example, if $\beta=0$, the class of all $f \in K$ of type less than $\pi$ is a uniqueness class for $\left\{\Delta^{n} f(0)\right\}$, but if $\beta=i$, the class of all $f \in K$ of type less than $\rho$ cannot be a uniqueness class for $\left\{\Delta^{n} f(\right.$ in $\left.)\right\}$ if

$$
\rho>|(-\log 2)+i \pi / 4| \approx .85,
$$

the modulus of the zero of $W^{\prime}$ nearest the origin.

For $\beta$ complex there is symmetry about the real axis, i.e., if $W_{\beta}(\zeta)$ $=e^{\beta \zeta}\left(e^{\zeta}-1\right)$, then

$$
\overline{W_{\bar{\beta}}(\bar{\zeta})}=\overline{e^{\overline{\beta \zeta}}\left(e^{\bar{\zeta}}-1\right)}=e^{\beta \zeta}\left(e^{\zeta}-1\right)=W_{\beta}(\zeta) .
$$


Thus if $W_{\beta}$ maps a set $\Omega_{\zeta}$ one to one onto a star shaped set, then $W_{\bar{\beta}}$ maps the conjugate of $\Omega_{\zeta}$ one to one onto a star shaped set. Thus we need consider only values of $\beta$ in the upper half plane. We give uniqueness and admissibility results for $\beta=c i$ and $\beta=-(1 / 2)+c i, c$ real.

1. $\beta=c i, c>0$. To determine a set $\Omega_{\zeta}$ on which $W(\zeta)=e^{c i \zeta}\left(e^{\zeta}-1\right)$ is univalent, we find the zeros of $W^{\prime}(\zeta)$ to be

$$
\zeta_{n}=\log \left(c / \sqrt{ }\left(1+c^{2}\right)\right)+i\left(\tan ^{-1}(1 / c)+2 n \pi\right) \quad(n=0, \pm 1, \pm 2, \cdots)
$$

where principal values of $\log$ and $\tan ^{-1}$ are taken. Thus all zeros of $W^{\prime}$ have real part $\log \left(c / \sqrt{ }\left(1+c^{2}\right)\right)<0$. If we let $w_{n}=W\left(\zeta_{n}\right)$, then $w_{n}=e^{-2 n c} w_{0}$, so that all $w_{n}$ have the same argument which is

$$
c \log \left(c / \sqrt{ }\left(1+c^{2}\right)\right)+\arg (-1+c i) .
$$

We denote this argument by $\alpha$.

Solving $\arg \left(e^{c i \xi}\left(e^{\zeta}-1\right)\right)=\alpha$, we obtain

$$
y=(\alpha-c x)-\sin ^{-1}\left(e^{-x} \sin (\alpha-c x)\right)
$$

for some choice of $\sin ^{-1}$ such that

$$
e^{x} \cos \left[\sin ^{-1}\left(e^{-x} \sin (\alpha-c x)\right)\right] \geqq \cos (\alpha-c x)
$$

as necessary and sufficient conditions on a point $\zeta=x+i y$ in order that its image have argument $\alpha$. Let $C_{0}$ denote the curve in the half plane $x \geqq \mathscr{R}\left(\zeta_{0}\right)$ given by (1) taking principal value of inverse sine. It can be shown that the points on $C_{0}$ satisfy (2), that $\zeta_{0}$ is on $C_{0}$, and that $C_{0}$ is mapped one to one onto the ray $r_{\alpha}=\left\{\rho e^{i \alpha}|\rho \geqq| w_{0} \mid\right\}$. Since $\arg W(\zeta-2 \pi i)=\arg W(\zeta)$ and $|W(\zeta-2 \pi i)|=e^{2 \pi c}|W(\zeta)|$, translating $C_{0}$ by $-2 \pi i$ gives a curve $C_{-1}$ which is mapped one to one onto $\left\{\rho e^{i \alpha}|\rho \geqq| w_{-1} \mid\right\}$. Since $W$ is entire, there exists a curve $T$ joining $\zeta_{0}$ and $\zeta_{-1}$ which is mapped one to one onto the line segment from $w_{0}$ to $w_{-1}$. It can be shown that the region bounded by $C_{0} \cup T \cup C_{-1}$ and containing the origin is mapped one to one onto the $w$ plane minus $r_{\alpha}$. We take this region as $\Omega_{5}$.

Since $C_{0}$ and $C_{-1}$ are asymptotic to $y=\alpha-c x$ and $y=\alpha-c x-2 \pi$ respectively as $x \rightarrow+\infty$, and $\zeta_{-1}$ on the boundary of $\Omega_{\zeta}$ is not contained in the strip between these asymptotes, $\Omega_{\zeta}$ is not convex. Since angles are doubled at $\zeta_{-1}$, the curves $C_{-1}$ and $T$ meet at right angles there. Graphing shows that $\Omega_{\zeta}$ resembles a lengthwise cross section of an upside down canoe of infinite length.

The set $\Omega_{w}^{\prime}$, the complement of the image of $\Omega_{\zeta}$ under $w=e^{c i \zeta}\left(e^{\zeta}-1\right)$ is the ray $\left\{w=\rho e^{i \alpha}|\rho \geqq| w_{0} \mid\right\}$; so we can apply the new form of Theorem 1 to conclude that given a sequence $\left\{b_{n}\right\}$ of complex numbers, there exists a function $f$ in $K\left[\Omega_{\zeta}\right]$ such that $\Delta^{n} f(c i)=b_{n}(n=0,1,2, \ldots)$ if and only if 


$$
b(z)=\sum_{n=0}^{\infty} b_{n} z^{n}
$$

is analytic at the origin and can be continued to the closed line segment from the origin to $w_{0}^{-1}$.

For example, if $\beta=i$, then $w_{0}^{-1}=\rho e^{i \theta}$ where $\rho=\exp ((\pi / 4)+(1 / 2) \log 2)$ $\approx 3.10$ and $\theta=(-3 \pi / 4)+\log 2$.

2. $\beta=(-1 / 2)+c i, c>0$. If $W(\zeta)=e^{((-1 / 2)+c i) \zeta}\left(e^{\zeta}-1\right)$, then $W^{\prime}\left(\zeta_{n}\right)=0$ for $\zeta_{n}=2 i\left(\tan ^{-1}(1 / 2 c)+n \pi\right)$, principal value of inverse tangent, $n=0, \pm 1$, $\pm 2, \cdots$. The points $\zeta_{n}$ are mapped by $W$ into points

$$
w_{n}=i\left\{2(-1)^{n}\left(1+4 c^{2}\right)^{(-1 / 2)} \cdot \exp \left[-2 c n \pi-2 c \tan ^{-1}(1 / 2 c)\right]\right\} .
$$

Solving $\mathscr{R}(W(x+i y))=0$, besides the solution $x=0$ which is not useful for our purpose, we obtain the relation

$$
y=2 \tan ^{-1}[\tanh (x / 2) \tan ((\pi / 2)-c x)] .
$$

It can be shown that for every choice of the value of inverse tangent, $\mathscr{R}(W(x+i y))$ does equal zero. If $(x, y)$ satisfies $(3)$, then $(-x, y)$ does also. Let $C_{0}$ be the curve satisfying (3), symmetric with respect to the imaginary axis, and for $x>0$ having inverse tangent chosen as follows:

Let $p$ be defined by $p \equiv(\pi / 2)-c x(\bmod 2 \pi)$ and $0 \leqq p<2 \pi$. For positive $x, 0<\tanh (x / 2)<1$. Therefore, for each $x>0$, there exists a unique number $q$ in the same quadrant as $p$ such that $\tan q=\tan [\tanh (x / 2) \tan p]$. Choose $\tan ^{-1}[\tanh (x / 2) \tan ((\pi / 2)-c x)]=(\pi / 2)-c x+(q-p)$. For $x=0$, choose $y$ to make $C_{0}$ continuous there.

Then $y$ is a continuous function of $x$ except possibly at values of $x$ for which $p=0, \pi / 2, \pi$, or $3 \pi / 2$. But, except for the case of $x$ approaching zero, as $p$ approaches any of these values from either side, $q$ approaches $p$, so $y$ is continuous at these points also. Since for $0<x<\pi / c$, principal value of inverse tangent was taken, and since $\lim _{\rightarrow 0}[\tanh (x / 2) \tan ((\pi / 2)-c x)]$ $=1 /(2 c)$, we have $y(0)=2 \tan ^{-1}(1 /(2 c))$; so $\zeta_{0}$ lies on $C_{0}$. The part $C_{0}^{+}$of $C_{0}$ in the right half plane contains no zeros of $W^{\prime}$ and since it maps into a ray, the mapping is one to one. Since $|W(x+i y)|$ increases unboundedly with $x$ for $x \geqq 0$, the image of $C_{0}^{+}$is $\left\{i y|y \geqq| w_{0} \mid\right\}$. We have $W(-\zeta)$ $=-W(\zeta)$, so the part of $C_{0}$ in the left half plane is mapped one to one onto the same ray. We also have $W(\zeta+2 n \pi i)=(-1)^{n} e^{-2 n \pi c} W(\zeta)$; so if we let $C_{-1}$ be the curve obtained by translating $C_{0}$ by $-2 \pi i$, then $C_{-1}$ is mapped two to one onto the ray $\left\{i y\left|y \leqq-e^{2 \pi c}\right| w_{0} \mid\right\}$.

We take the region containing the origin and bounded by $C_{0} \cup C_{-1}$ as $\Omega_{5}$. It can be shown that this region is mapped one to one onto the $w$ plane minus the parts of the imaginary axis from $w_{0}$ to $i \infty$ and from $w_{-1}$ to $-i \infty$. Since $C_{0}$ is asymptotic to $y=\pi-2 c|x|$ and $C_{-1}$ is asymptotic to $y=-\pi-2 c|x|$, 
$\Omega_{\zeta}$ is not convex. It resembles a cross section of a metal rod of infinite length bent over a person's knee.

Again, we obtain immediately that $K\left[\Omega_{\zeta}\right]$ is a uniqueness class for

$$
\left\{\Delta^{n} f((-1 / 2)+c i)\right\}
$$

and that for a given sequence $\left\{b_{n}\right\}$ of complex numbers, there exists a function $f$ in $K\left[\Omega_{\zeta}\right]$ such that $\Delta^{n} f((-1 / 2)+c i)=b_{n}(n=0,1,2, \cdots)$, if and only if the function $b(z)=\sum_{n=0}^{\infty} b_{n} z^{n}$ is analytic at the origin and can be continued to the closed interval of the imaginary axis from $w_{0}^{-1}$ to $w_{-1}^{-1}$. For example, if $c=1 / 2$, this is the interval from $-(1 / \sqrt{ } 2) e^{\pi / 4} i$ to $(1 / \sqrt{ } 2) e^{-3 \pi / 4} i$ which is approximately the interval $[-1.54 i, .067 i]$.

3. Lidstone functionals. The Lidstone functionals given by $\mathscr{L}_{2 n}(f)=f^{(2 n)}(0)$ and $\mathscr{L}_{2 n+1}(f)=f^{(2 n)}(1)(n=0,1,2, \ldots)$ do not have a representation (1.1), so that the theorems used up to this point do not apply, but the methods used in proving those theorems can be modified to obtain uniqueness and admissibility results. If $\Omega_{\zeta}$ is taken as the $\zeta$ plane cut from $\pi i$ to $i \infty$ and from $-\pi i$ to $-i \infty$ along the imaginary axis, then $K\left[\Omega_{\zeta}\right]$ is a uniqueness class for $\left\{\mathscr{L}_{n}\right\}[2]$. Since the set $\Omega_{\zeta}$ is not convex, the admissibility result for $\left\{\mathscr{L}_{n}\right\}$ and $K\left[\Omega_{\zeta}\right]$ obtained previously [4], gave only a necessary condition. To obtain a sufficient condition, it was possible to deal only with sets $C_{\zeta}$ which were convex subsets of $\Omega_{\zeta}$ having the property that $z \in C_{\zeta}$ implies $(-z) \in C_{\zeta}$. By making the same modification in the proof as was made in Theorem 1 , the hypothesis of convexity of $C_{\zeta}$ can again be removed and we can give the following necessary and sufficient condition for admissibility for $\left\{\mathscr{L}_{n}\right\}$ and $K\left[\Omega_{\zeta}\right]$ :

Given a sequence $\left\{b_{n}\right\}$ of complex numbers, there exists a function $f \in K\left[\Omega_{5}\right]$ such that $f^{(2 n)}(0)=b_{2 n}, f^{(2 n)}(1)=b_{2 n+1}(n=0,1,2, \ldots)$, if and only if the function $b(z)=\sum_{n=0}^{\infty} b_{n} z^{n}$ is analytic at the origin and can be continued to the interval $[-i / \pi, i / \pi]$ of the imaginary axis.

Proof of sufficiency. Let $b(z)$ (again defined by $\sum_{n=0}^{\infty} b_{n} z^{n}$ and its analytic continuation) be regular on a simply connected domain $R$ containing $[-i / \pi, i / \pi]$. Let

$$
f(z)=\frac{1}{2 \pi i} \int_{E} \frac{b(t)}{t}\left[\frac{\sinh [(1-z) / t]}{\sinh (1 / t)}+\frac{\sinh (z / t)}{t \sinh (1 / t)}\right] d t
$$

where $E$ is a simple contour contained in $R$, encloses the interval $[-i / \pi, i \pi]$, and is symmetric with respect to the origin. This is possible since $[-i / \pi, i / \pi]$ is symmetric with respect to the origin. Then $f^{(2 n)}(0)=b_{2 n}$ and $f^{(2 n)}(1)$ $=b_{2 n+1}(n=0,1,2, \ldots)[4]$. We can write

$$
f(z)=\frac{1}{2 \pi i} \int_{E} \frac{b(t)}{2 t \sinh (1 / t)}\left[e^{(1-z) / t}-e^{-(1-z) / t}+e^{z / t} / t-e^{-z / t} / t\right] d t
$$


and, as in the proof of Theorem 1 , for $\mathscr{R}(\zeta)$ sufficiently large,

$$
\begin{aligned}
F(\zeta) & =\frac{1}{2 \pi i} \int_{E} \frac{b(t)}{2 t \sinh (1 / t)} \int_{0}^{\infty} e^{-s \zeta}\left[e^{(1-s) / t}-e^{-(1-s) / t}+e^{s / t} / t-e^{-s / t} / t\right] d s d t \\
& =\frac{1}{2 \pi i} \int_{E} \frac{b(t)}{2 t \sinh (1 / t)}\left[\frac{e^{1 / t}}{\zeta+1 / t}-\frac{e^{-1 / t}}{\zeta-1 / t}+\frac{1}{t(\zeta-(1 / t))}-\frac{1}{t(\zeta+(1 / t))}\right] d t,
\end{aligned}
$$

which gives the analytic continuation of $F$ to any simply connected region, containing the point at infinity, on which the integral exists. Since $E$ encloses $[-i / \pi, i / \pi], t$ and $\sinh (1 / t)$ are nonzero for all $t \in E$. Therefore, the integral exists for all $\zeta$ such that for all $t \in E, \zeta \neq \pm 1 / t$. Since $E$ is symmetric with respect to the origin, this is the set of all $\zeta$ for which for all $t \in E, \zeta \neq 1 / t$. If $\zeta \in \Omega_{\zeta}^{\prime}$, then $(1 / \zeta) \in[-i / \pi, i / \pi]$, so that $\zeta \neq 1 / t$ for every $t \in E$. Thus $F$ is regular on $\Omega_{5}^{\prime}$ or $f \in K\left[\Omega_{5}\right]$.

\title{
BibLIOGRAPHY
}

1. R. P. Boas and R. C. Buck, Polynomial expansion of analytic functions, Springer-Verlag, Berlin, 1958.

2. R. C. Buck, Interpolation series, Trans. Amer. Math. Soc. 64 (1948), 283-298.

3. , Integral valued entire functions, Duke Math. J. 15 (1948), 879-891.

4. R. F. DeMar, Existence of interpolating functions of exponential type, Trans. Amer. Math. Soc. 105 (1962), 359-371.

5. A. O. Gel'fond, Interpolation et unicité des fonctions entières, Mat. Sb. (N.S.) 46 (1938), 115-147.

\author{
Miami UnIVersity, \\ OXFORD, OHIO \\ National Bureau of Standards, \\ Washington, D. C.
}

